\title{
Age and sex related differences in shoulder abduction fatigue
}

\author{
John D. Collins ${ }^{1}$ and Leonard O'Sullivan ${ }^{2^{*}}$ (D)
}

\begin{abstract}
Background: Injury prevalence data commonly indicate trends of higher rates of work-related musculoskeletal disorders in older workers over their younger counterparts, and for females more than males. The purpose of this study was to investigate age and sex-related differences in manifestations of shoulder muscle fatigue in a cohort of young and older working age males and females, in a single experiment design allowing for direct comparison of the fatigue effects between the target groups.

Methods: We report upper trapezius muscle fibre Conduction Velocity (CV) as an indicative measure of muscle fatigability, and isometric endurance time, at three levels of shoulder abduction lifting force set relative to participants' maximal strength.

Results: Upper trapezius conduction velocity was significantly different between the young and old groups $(p=0$. $002)$ as well as between males and females $(p=0.016)$. Shoulder abduction endurance time was affected by age $(P=0.024)$ but not sex $(p=0.170)$.

Conclusions: The study identified age-related improvement in muscle fatigue resistance and increased resistance for females over males, contrary to injury prevalence trends. The muscle fatigue effects are most likely explained by muscle fibre type composition. Experimental fatigue treatments of the upper trapezius were tested at exposures relative to the participants' strength. Absolute strength is higher when young and is generally higher for males. The findings of this study point towards age and sex-related differences in strength rather than in muscle fatigue resistance as a primary cause for the differences in the injury trends.
\end{abstract}

Keywords: Musculoskeletal disorders, Age, Sex, Trapezius, Shoulder, Fatigue

\section{Background}

Occupational injury and symptom prevalence data often indicate higher rates for older workers and for females $[1$, 2]. Collins and O'Sullivan [3] previously reported prevalence of neck/shoulder symptoms of Musculo-Skeletal Disorders (MSDs) for young and old age groups in a sedentary occupation, a trend also described by others [4]. In the study by Collins and O'Sullivan symptoms were highest for the oldest cohort and for females compared to their male counterparts. Silvia et al. [5] reported back pain across multiple industrial sectors and again reported higher prevalence for females. Laperriere et al. [6] detailed higher prevalence of self-reported work-related pain for females than males in food service work. Anton and

\footnotetext{
* Correspondence: leonard.osullivan@ul.ie

${ }^{2}$ School of Design and Health Research Institute, University of Limerick, Limerick, Ireland

Full list of author information is available at the end of the article
}

Weeks [7] described higher rates of MSD symptoms for female grocery workers than their male counterparts. In addition, they reported higher rates for workers aged 35+ years compared to younger age groups. Regarding ageing and MSDs, Slovak et al. [8] analysed data from The Health and Occupational Reporting Network which indicated a fivefold increase in work-related musculoskeletal disorders from ages 15-24 to 45-64. This is of considerable concern in view of the ageing workforce [9]. Many occupational health and ergonomics studies of MSD prevalence focus on heavy manual work, yet MSDs are highly prevalent in low force sedentary work, specifically in relation to the shoulder, for example in computer-based work $[2,4,10]$.

The authors propose that there are three primary explanations for age and sex-related differences in muscle-related occupational injuries. The first explanation is due to differences in exposures, where some occupational

(c) The Author(s). 2018 Open Access This article is distributed under the terms of the Creative Commons Attribution 4.0 International License (http://creativecommons.org/licenses/by/4.0/), which permits unrestricted use, distribution, and reproduction in any medium, provided you give appropriate credit to the original author(s) and the source, provide a link to the Creative Commons license, and indicate if changes were made. The Creative Commons Public Domain Dedication waiver (http://creativecommons.org/publicdomain/zero/1.0/) applies to the data made available in this article, unless otherwise stated. 
groups with higher risk of developing MSDs are dominated by one sex and/or age group. Controlling for exposure, a second potential explanation of variations are age/sex-related differences in muscle strength. A third possible explanation is due to differences in muscle fibre composition. The current study focuses on evaluating the latter explanation by measuring muscle fibre conduction velocity in a group of old and young participants.

Muscle activity, particularly where forceful exertions exist, has been implicated in many studies as a primary risk factor of muscle fatigue $[11,12]$. However, muscle fatigue is not limited to high force contractions as low force static contractions can also cause muscle fatigue [13]. It is therefore important to acknowledge age and sex-based differences in fibre distribution as these characteristics may explain, at least in part, injury prevalence patterns detailed in previous research [3]. If age and sex-related differences in fibre types affect endurance and fatigue, it may be postulated that the magnitude of differences is important from an injury causation perspective.

There is good reason to consider muscle fibre-related differences as a mechanism of increased injury, especially for low force static contractions. The scientific literature on muscle fatigue resistance $[14,15]$ and muscle fibre type composition indicate age [16] and sex-based differences [17]. Furthermore, studies have investigated muscle fatigue and function specifically for low force muscle activity contractions, which is particularly important considering muscle fibre type differences $[12,13,17]$. According to the Hennmann Principle [18] and the Cinderella hypothesis [19], sustained low-level isometric contractions set up a stereotyped recruitment pattern of Motor Units (MUs) according to the size principle. Low threshold (type I) MUs are constantly active even in situations of continuous low muscle activity which could result in metabolically overloaded 'Cinderella' muscle fibres.

There are numerous studies of age and sex-related differences in muscle performance [14, 15]. However, we have been unable to find any studies specifically assessing age and sex-based differences in muscle fibre conduction velocity of the trapezius. The aim of this study was to investigate manifestations of shoulder muscle fatigue across age and sex. Conduction Velocity (CV) was used as an index of muscle fatigue in this experiment. It is possibly the most accurate physiological parameter of muscle fatigue as it is affected by fibre size and changes in $\mathrm{pH}$, whereby $\mathrm{CV}$ values decrease during fatigue contractions and the slope of the $\mathrm{CV}$ values is indicative of the rate of fatigue in the muscle [20].

\section{Method}

\section{Study design and statistical analysis}

This was a laboratory based study of upper trapezius muscle fibre Conduction Velocity (CV) (as a measure of muscle fatigue and endurance) for three relative levels of shoulder abduction, tested with the shoulder abducted $90^{\circ}$. The two dependent variables were CV (normalised slope) and Endurance Time (ET). The independent variables were sex, age (young and old) and shoulder abduction exertion load (0, 10 and 20\% Maximum Voluntary Contraction $(\mathrm{MVC}))$. The exertion levels were based on previous studies of shoulder fatigue [21]. $\mathrm{VO}_{2} \mathrm{MAX}$ was entered as a covariate to control for differences in aerobic fitness.

\section{Participants}

There were 40 participants, 20 males and 20 females, with 10 each in the young and old sex-age groups (young participants mean age 26.0 years \pm 2.18 SD; older group mean age 59.6 years $\pm 3.17 \mathrm{SD}$ ). A power analysis indicated this sample would yield an experimental power of $>0.8$.

The experiment was approved by the University of Limerick Research Ethics Committee. Participants were recruited through advertisements on the University campus and through requests for volunteers through fellow researchers' contacts. Participants gave their written informed consent prior to testing. No participant reported any known symptoms of locomotive or musculoskeletal disorders.

\section{Equipment}

As muscle $\mathrm{CV}$ reduces with time in fatiguing muscles, the slope of the data, normalised to the initial value of the treatment, was selected as the index of fatigue [20]. $\mathrm{CV}$ was measured using a disposable 16-array surface electrode at a $5 \mathrm{~mm}$ electrode pitch (Model ELSCH016 electrode, OT Bioelettronica). Signals were sampled by a multichannel EMG amplifier (OT Bioelettronica, Torino, Italy). The EMG signals were amplified, band pass filtered ( $3 \mathrm{~dB}$ bandwidth, $10-500 \mathrm{~Hz}$, roll-off of $40 \mathrm{~dB} /$ decade), sampled at $2048 \mathrm{~Hz}$, and stored on a PC (12 bit $\mathrm{A} / \mathrm{D}$ converter). $\mathrm{VO}_{2}$ MAX was measured using a portable $\mathrm{O} 2$ analyser (Model Cosmed $\mathrm{k} 4 \mathrm{~B}^{2}$ ) and a cycle ergometer to perform the aerobic activity.

Shoulder abduction exertion was measured using a commercial force meter (Mecmesin Advanced Force Gauge AFG-500 N) modified with an adjustable level handle attached to a platform.

\section{Procedure}

\section{Part 1 shoulder abduction MVC}

The testing posture involved the participant seated, with feet firmly on the ground and their back in an upright posture, the dominant shoulder abducted to $90^{\circ}$, the elbow fully extended, and the forearm fully pronated. The handle of the force meter was adjusted to the required testing posture. Participants performed an initial warm-up which consisted of a number of repetitive shoulder movements from $0^{0}$ to $90^{\circ}$ abduction. Instructions to the participants informed them to abduct their 
Table 1 Summary of ANOVA/ANCOVA main and interaction effects on endurance time

\begin{tabular}{|c|c|c|c|c|c|c|c|}
\hline \multirow[t]{2}{*}{ Dependent variable } & \multicolumn{3}{|c|}{ Main Effects } & \multicolumn{4}{|c|}{ Interaction } \\
\hline & $\mathrm{L}$ & $S$ & A & AxS & $\mathrm{AxL}$ & SXL & $\mathrm{A} \times S \times L$ \\
\hline Endurance Time & $0.0005^{* *}$ & 0.1 & $0.021^{*}$ & 0.491 & 0.521 & 0.15 & $0.012^{*}$ \\
\hline Endurance Time ( $\mathrm{VO}_{2} \mathrm{MAX}$ as covariate) & $0.0001^{* *}$ & 0.175 & $0.024^{*}$ & 0.308 & 0.548 & 0.242 & $0.009^{* *}$ \\
\hline
\end{tabular}

$\mathrm{L}=$ Load (exertion level) $\mathrm{S}=\mathrm{Sex} \mathrm{A}=$ Age

${ }^{*} p<0.05{ }^{* *} p<0.01$

pronated arm while grasping the handle of the force meter, to generate their maximum lifting effort and sustaining it for $3 \mathrm{sec}$, in line with the Caldwell regime [22]. Three trials were conducted with 5 min of rest between each treatment. The maximum result was determined as the participant's MVC.

\section{Part 2 upper trapezius CV and shoulder abduction endurance}

The participants' skin was shaven, if required, and prepared with abrasive paste and alcohol wipes to reduce skin impedance [23]. Conductive gel was injected into each space of the array electrode and secured to the skin with tape. The electrode was positioned over the upper trapezius lateral to the innervation zone, between the seventh cervical vertebra and the posterior tip of the acromion. The Innervation Zone (IZ) of the muscle was detected using a bar electrode and the position marked on the skin to position the array.

Each participant completed three static shoulder abduction endurance exertions at 0,10 , and 20\% MVC. Contractions were maintained until the participant could no longer sustain the exertion. The target percentage MVC levels were calculated by the experimenter and the participants were instructed to exert the force levels visually via the real-time values on the force meter interface. The $0 \%$ MVC exertion involved holding the arm outright without exerting a lifting force on the force meter.
The treatments were randomised for each participant using Latin Square orders, which are unique orders of treatments for each participant. A 10-min resting period followed each endurance test with additional rest given if residual fatigue or discomfort was reported, as per Yassierli and Nussbaum [24].

\section{Part 3 post-test measurements of the covariate $\mathrm{VO}_{2} \mathrm{MAX}$}

The starting workload was estimated based on age and level of activity. The cadence was adjusted to achieve a heart rate of $60-85 \%$ of maximal capacity (i.e. 220-age) and $\mathrm{VO}_{2}$ MAX was measured in real time using the Cosmed software system.

\section{Statistical analysis}

Data were presented as means and Standard Deviation (SD) of the mean. Statistical significance was set at $p<0.05$. Statistical Analysis was performed using SPSS V 22. The Kolmogorov-Smirnov test applied to the data indicated the shoulder abduction MVC values were normally distributed, but the CV and ET data were not. The log transformation was successful in normalising these data. Independent samples t-tests were used to compare the MVC data between the young versus old age groups, and separately for males versus females. ANOVA was used to test the main and interaction effects on both $\mathrm{CV}$ and ET, and repeated with ANCOVA to include $\mathrm{VO}_{2}$ max as the covariate.

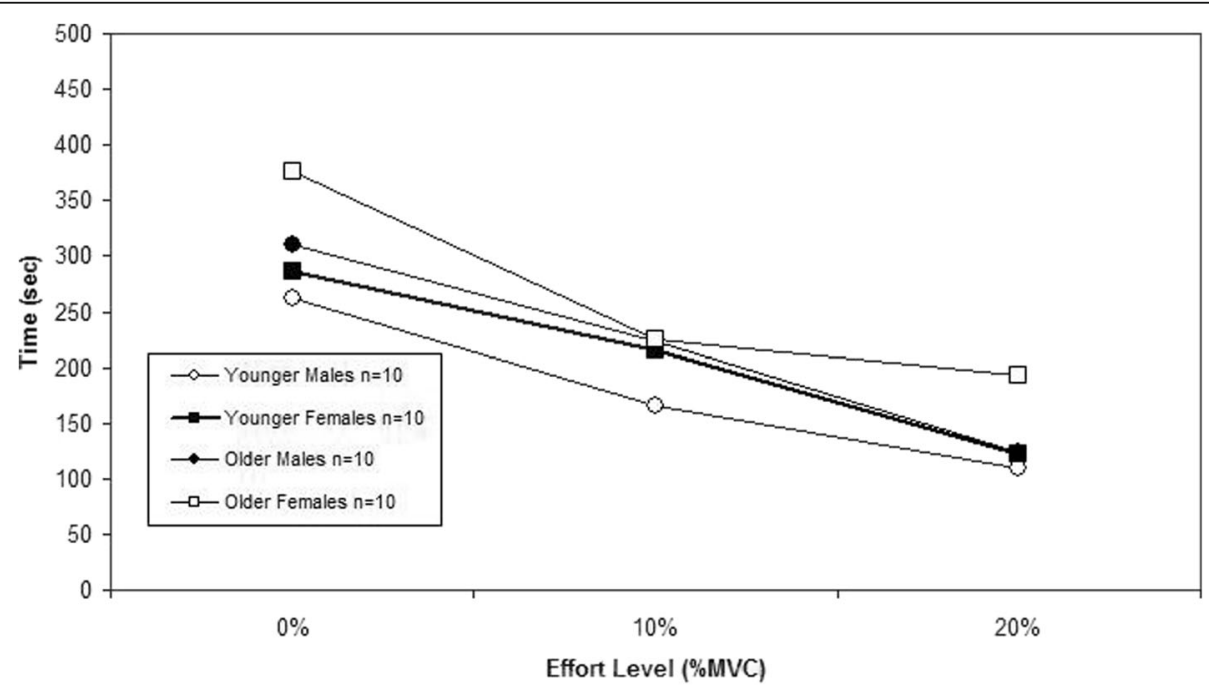

Fig. 1 Mean endurance times illustrating the statistically significant effects for Load, Age, and the Age $\times$ Sex $x$ Load interaction $(p<0.05)$ 
Table 2 Summary of ANOVA/ANCOVA main and interaction effects on normalised rate of change of CV

\begin{tabular}{|c|c|c|c|c|c|c|c|}
\hline \multirow[t]{2}{*}{ Dependent variable } & \multicolumn{3}{|c|}{ Main Effects } & \multicolumn{4}{|c|}{ Interaction } \\
\hline & $\mathrm{L}$ & $S$ & A & AxS & $\mathrm{AxL}$ & SXL & $A \times S \times L$ \\
\hline Conduction Velocity & $0.049^{*}$ & $0.035^{*}$ & $0.003^{*}$ & 0.621 & $0.044^{*}$ & 0.187 & 0.845 \\
\hline Conduction Velocity $\left(\mathrm{VO}_{2} \mathrm{MAX}\right.$ as a covariate) & 0.13 & $0.016^{*}$ & $0.002^{* *}$ & 0.292 & $0.035^{*}$ & 0.154 & 0.827 \\
\hline
\end{tabular}

$\mathrm{L}=$ Load $\mathrm{S}=\mathrm{Sex} \mathrm{A}=$ Age

${ }^{*} p<0.05{ }^{* *} p<0.01$

\section{Results}

Mean shoulder abduction MVC of the young group was $73.1 \mathrm{~N} \pm(27.30 \mathrm{SD})$ and mean MVC of the older individuals was $64.01 \mathrm{~N} \pm(24.15 \mathrm{SD})$. This difference was not statistically significant. Mean shoulder abduction MVC for males was $87.8 \mathrm{~N}( \pm 21 \mathrm{SD})$ and for females $49.3 \mathrm{~N}( \pm 12.3$ $\mathrm{SD})$, which were significantly different (t-test $p=0.0001$ ).

Regarding endurance times, Table 1 details the results of the statistical analysis while Fig. 1 depicts the plots of the mean values by age, sex and load. Age had a highly significant effect on Endurance Time $(p=0.02)$, with higher times for the older age group. $T$ there was no significant effect $(p=0.1)$ for sex. The three-way interaction was also significant $(p=0.01)$.

Regarding the upper trapezius conduction velocity data, Table 2 details the results of the statistical analysis while Table 3 and Fig. 2 detail the mean and standard deviation of the data for the experimental conditions. Age had a significant effect $(p=0.002)$ with greater slopes (higher fatigue) for the younger groups. Sex also had a significant effect $(p=0.016)$ with males showing greater slopes (higher fatigue). There was a significant Age $\mathrm{x}$ Load interaction $(p=0.035)$.

\section{Discussion}

The principal findings of this study are an apparent age-related improvement in muscle endurance and increased fatigue resistance for females over males. These trends have been previously observed in individual studies of age or sex for muscles groups. There are two key strengths to this study over previous fatigue studies of this nature. Firstly, it is a single experimental study of the same fatigue conditions involving both males and females of both age groups. This enables a direct comparison of the fatigue

Table 3 Mean endurance times and SD by load age and sex

\begin{tabular}{|c|c|c|c|c|c|}
\hline \multirow{2}{*}{$\begin{array}{l}\text { Load } \\
\text { (Exertion level) }\end{array}$} & \multirow[t]{2}{*}{ Group } & \multicolumn{2}{|l|}{ Male } & \multicolumn{2}{|c|}{ Female } \\
\hline & & Mean & SD & Mean & SD \\
\hline \multirow[t]{2}{*}{ 0\% MVC } & Young & 262 & 99 & 285 & 145 \\
\hline & Older & 310 & 149 & 377 & 168 \\
\hline \multirow[t]{2}{*}{$10 \% \mathrm{MVC}$} & Young & 166 & 66 & 215 & 109 \\
\hline & Older & 224 & 80 & 225 & 34 \\
\hline \multirow[t]{2}{*}{$20 \%$ MVC } & Young & 109 & 31 & 122 & 48 \\
\hline & Older & 124 & 39 & 193 & 25 \\
\hline
\end{tabular}

differences between these groups, which, to our knowledge, is not present in the literature for working age adults. The second key strength of this study is the measurement of muscle fatigue via muscle fibre conduction velocity for the experimental fatigue conditions tested, and for a shoulder muscle commonly associated with MSDs.

The age-related improvement in fatigue identified in this study may be due to differences in muscle fibre type compositions [25]. Aged muscles have been characterised as muscles with a type I fibre dominance [26] resulting in increased fatigue resistance [27]. Type I fibre fatigue resistance is indicated to be due to their myosin heavy chain cross bridges $[28,29]$. Merletti et al. [30] detail that reduction in the motor unit firing rate also plays a role in improvement of age-related muscle fatigue resistance. Muscle fibre type differences also most likely explain the increased fatigue resistance for females. Although there is a similar distribution of fast and slow fibre types for males and females, there is, however, a significant sex-related difference in the total area occupied by type I fibres [28, 31], which increases oxidative capacity of these fibres, increasing fatigue resistance for female muscle. Fulco et al. [32] propose that female muscle has increased muscle oxidative phosphorylation, while Crowther and Gronka [33] have identified that the muscle fibres recruited first in voluntary contractions have a higher oxidative capacity than those recruited last. Sex-based differences have also been observed in muscle function [28], but explanations for these differences remain underdeveloped. In accordance with the Henneman Theory and the Cinderella Hypothesis, female muscle would contain a greater oxidative capacity prolonging fatigue. Each of these suggestions may explain the sex-related difference in the current study, however, additional tests incorporating greater loads would be advantageous in supplementing these attributes.

This study reinforces the need for clinicians and policymakers to emphasise workplace and policy-level occupational health strategies to correct the clear trends of elevated work-related injuries/symptoms for certain groups of workers. This can be achieved through group level exposure monitoring, and/or through improved workplace risk assessments/monitoring sensitivity of more vulnerable workers.

A weakness of the study is the sample size. Each group contained only ten participants, but it should be noted that this is greater than the full sample size of many other EMG-based lab studies. The sample sizes were 


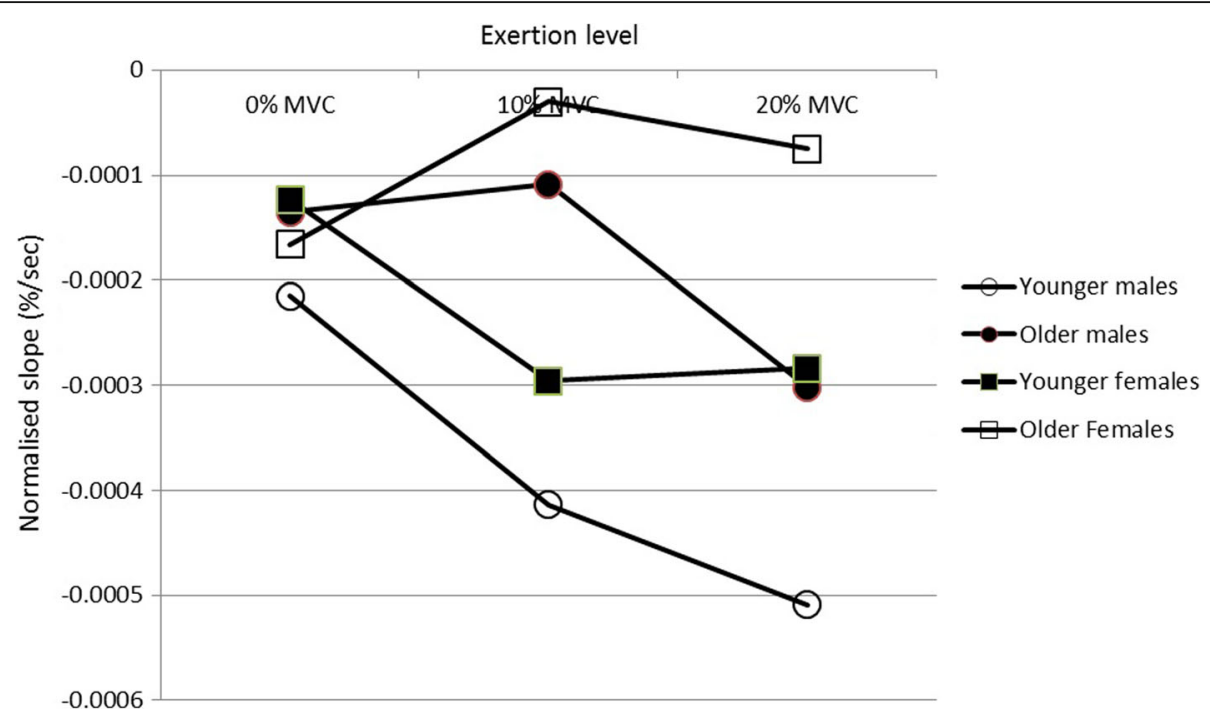

Fig. 2 Mean normalised muscle fibre conduction velocities for the upper trapezius, by Load, Age and Sex

considered adequate for statistical analysis in this experiment. However, a larger sample would clarify borderline near significant results, which may be indicative of type II errors. The testing posture, while typical of static arm positions in many construction and industrial tasks, may not represent many typical arm postures involving low levels of shoulder flexion/abduction, for example in computer work, also representing a limitation of the study. The mean age of the older participants was 59 years, which is not representative of the older general population, however it is representative of the older working age population where many people retire by the age of 65 years. Further studies should be performed with older participants than studied in this experiment to assess the wider generalisability of these findings.

\section{Conclusions}

The overall conclusion from this research was that the older cohort exhibited decreased shoulder strength, longer endurance times, and signs of slower progression of muscular fatigue, suggesting type I fibre dominance in aged muscles. Females exhibited higher endurance times and slower progression of muscular fatigue than the males. The importance of this work is that it identified that the groups with increased fatigue resistance (older age \& females) are indicated to be those typically with lower muscle strength in the working population. A key inference from the study is that when controlling for exposure, the trends in age and sex-related differences in shoulder MSD prevalence might not primarily be due to muscle fibre type related differences, but rather differences in muscle strength.
Availability of data and materials

The datasets used and/or analysed during the current study are available from the corresponding author on reasonable request.

\section{Authors' contributions}

JC was a Ph.D. researcher on this study. He planned, performed and analysed the study, and drafted the paper. LOS was the supervisor of the research. He assisted in the planning and oversight of the research, and provided feedback on the manuscript. Both authors read and approved the final manuscript.

Ethics approval and consent to participate

This research was approved by the Research Ethics Committee of the University of Limerick. All participants gave their written informed consent prior to testing.

Consent for publication

Not applicable.

Competing interests

The authors declare that they have no competing interests.

\section{Publisher's Note}

Springer Nature remains neutral with regard to jurisdictional claims in published maps and institutional affiliations.

\section{Author details}

${ }^{1}$ School of Design, University of Limerick, Limerick, Ireland. ${ }^{2}$ School of Design and Health Research Institute, University of Limerick, Limerick, Ireland.

Received: 14 October 2017 Accepted: 13 July 2018

Published online: 07 August 2018

References

1. Davis K, Dunning K, Jewell G, Lockey J. Cost and disability trends of workrelated musculoskeletal disorders in Ohio. Occup Med (Lond). 2014;64:608-15.

2. Ekman A, Andersson A, Hagberg M, Hjelm EW. Gender differences in musculoskeletal health of computer and mouse users in the Swedish workforce. Occup Med (Lond). 2000;50:608-13.

3. Collins JD, O'Sullivan L. Musculoskeletal disorder prevalence and psychosocial risk exposures by age and gender in a cohort of office based employees in two academic institutions. Int J Ind Ergon. 2015;46:85-97.

4. Janwantanakul $P$, Pensri $P$, Jiamjarasrangsri $V$, Sinsongsook T. Prevalence of self-reported musculoskeletal symptoms among office workers. Occup Med (Lond). 2008;58:436-8. 
5. Silvia C, Barros C, Cunha L, Carnide F, Santos M. Prevalence of back pain problems in relation to occupational group. Int J Ind Ergon. 2016;52:52-8.

6. Laperriere E, Messing K, Bourbonnais R. Work activity in food service: the significance of customer relations, tipping practices and gender for preventing musculoskeletal disorders. Appl Ergon. 2017;58:89-101.

7. Anton D, Weeks DL. Prevalence of work related musculoskeletal symptoms among grocery workers. Int J Ind Ergon. 2016;54:139-45.

8. Slovak A, Carder M, Money A, Turner S, Agius R. Work-related musculoskeletal conditions: evidence from the THOR reporting system 2002-2005. Occup Med (Lond). 2009:59:447-53.

9. Algarni FS, Gross DP, Senthilselvan A, Battie MC. Ageing workers with workrelated musculoskeletal Injuries. Occup Med (Lond). 2015;65:229-37.

10. Moen BE, Wieslander G, Bakke JV, Norbäck D. Subjective health complaints and psychosocial work environment among university personnel. Occup Med (Lond). 2013;63:38-44.

11. Li ZZ, Zhang WK, Ma W, L. Chen Z. Muscular fatigue and maximum endurance time assessment for male and female industrial workers. Int J Ind Ergon. 2014:44:292-7.

12. Troiano A, Naddeo F, Sosso E, Camarota G, Merletti R, Mesin L. Assessment of force and fatigue in isometric contractions of the upper trapezius muscle by surface EMG signal and perceived exertion scale. Gait Posture. 2008;28: 179-86.

13. Farina D, Falla D. Estimation of muscle fibre conduction velocity from twodimensional surface EMG recordings in dynamic tasks. Biomedical Signa Processing and Control. 2008;3:138-44.

14. Sundberg C, Kuplic A, Hassanlouei H, Hunter S. Mechanisms for the agerelated increase in fatigability of the knee extensors in old and very old adults. J Appl Physiol. 2018;91:2686-94.

15. Deering R, Senefeld J, Pashibin T, Hunter S. Muscle function and fatigability of trunk flexors in males and females. Biol Sex Differ. 2017;8:1-12.

16. Avin $K G$, Frey Law LA. Age-related differences in muscle fatigue vary by contraction type: a meta-analysis. J Am Phys Ther Assoc. 2011;91:1153-65.

17. Zhanga Z, Li Way K, Zhang W, Ma L, Chen Z. Muscular fatigue and maximum endurance time assessment for male and female industrial workers. Int J Ind Ergon. 2014;44:292-7.

18. Henneman E, Somjen G, Carpenter DO. Functional significance of cell size in spinal motoneurons. J Neurophysiol. 1965;28:560.

19. Hägg G. Static work loads and occupational myalgia-a new explanation model. In: Anderson P, Hobart D, Danoff, Electromographical kinesiology. Amsterdam: Elsevier; 1999.

20. Merletti R, Parker P. Electromyography: physiology, engineering and noninvasive applications. New York: Wiley-IEEE Press; 2004.

21. Wahlstrom J. Ergonomics, musculoskeletal disorders and computer work. Occup Med (Lond). 2005;55:168-76.

22. Caldwell L, Chaffin D, Dukes Dobos F, Kroemer K, Labach L, Snook S, Wasserman D. A proposed standard procedure for static muscle strength testing. Am Ind Hyg Assoc J. 1974;35:201-6.

23. Hermens HJ, Freriks B, Disselhorst-Klug C, Raru G. Development of recommendations for SEMG sensors and sensor placement procedures. J Electromyogr Kinesiol. 2000;10:361-74.

24. Yassierli. Nussbaum. MA., Muscle fatigue during intermittent isokinetic shoulder abduction: Age effects and utility of electromyographic measures. Ergonomics. 2007; 50; 1110-1126.

25. Trappe S, Gallagher P, Harber M, Carrithers J, Fluckey J, Trappe T. Single muscle fibre contractile properties in young and old men and women. $J$ Physiol. 2003;552:47-58.

26. Nilwirk R, Snijders T, Leenders M, Groen BB, van Kranenburg J, Verdijk LB, van Loon $\mathrm{L}$. The decline in skeletal muscle mass with aging is mainly attributed to a reduction in type II muscle fibre size. Exp Gerontol. 2013;48:492-8,

27. Merletti R, Benvenuti F, Doncarli C, Disselhorst-Klug C, Ferrabone R, Hermens H, Kadefors R, Lãubli T, Orizio C, Sjã-gaard G, Zazula D. The European project -neuromuscular assessment in the elderly worker: achievements in electromyogram signal acquisition, modelling and processing. Med Biol Eng Comput. 2004;42:429-31.

28. Yu F, Hedström M, Cristea A, Dalén N, Larsson L. Effects of ageing and gender on contractile properties in human skeletal muscle and single fibres. Acta Physiol. 2007;190:229-41.

29. Callahan DM, Miller MS, Sweeny AP, Tourville TW, Slauterbeck JR, Savage PD, Maugan DW, Ades PA, Beynnon BD, Toth MJ. Muscle disuse alters skeletal muscle contractile function at the molecular and cellular levels in older adult humans in a sex-specific manner. J Physiol. 2014;592:4555-73.
30. Merletti R, Farina D, Gazzoni M, Schieroni MP. Effect of age on muscle functions investigated with surface electromyography. Muscle Nerve. 2002;25:65-76.

31. Staron RS, Hagerman FC, Hikida RS, Murray TF, Hoster DP, Crill MT, Ragg KE, Toma K. Fiber type composition of the vastus Lateralis muscle of young men and women. Journal of Histochemistry Cytochemistry. 2000;48:623-30.

32. Fulco CS, Rock PB, Muza SR, Lammi E, Braun B, Cymerman A, Moore LG, Lewis SF. Gender alters impact of hypobaric hypoxia on adductor pollicis muscle performance. J Appl Physiol. 2001;91:100-8.

33. Crowther CG, Crowther GJ, Gronka RK. Fiber recruitment affects oxidative recovery measurements of human muscle in vivo. Med Sci Sports Exerc 2002;34:1733-7.
Ready to submit your research? Choose BMC and benefit from:

- fast, convenient online submission

- thorough peer review by experienced researchers in your field

- rapid publication on acceptance

- support for research data, including large and complex data types

- gold Open Access which fosters wider collaboration and increased citations

- maximum visibility for your research: over $100 \mathrm{M}$ website views per year

At $\mathrm{BMC}$, research is always in progress.

Learn more biomedcentral.com/submissions 